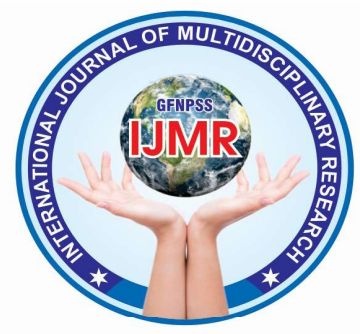

\title{
ASSESS THE EFFECTIVENESS OF AWARENESS PROGRAMME ON KANGAROO MOTHER CARE AMONG B.Sc. NURSING STUDENTS AT SELECTED NURSING COLLEGES SRI GANGANAGAR, RAJASTHAN
}

\author{
DR. ROBBY SOLANKI ${ }^{1}$, MR. SHAKTI SINGH SONI ${ }^{2}$, MS. JYOTSNA ${ }^{3}$ \\ ${ }^{1}$ Professor cum Principal (H.O.D. O.B.G. Nursing) S.N. College of Nursing, Sri Ganganagar. \\ ${ }^{2}$ Associate Professor cum H.O.D. Mental Health Nursing, S.N. College of Nursing, Sri Ganganagar. \\ ${ }^{3}$ Assistant Professor cum H.O.D. Child Health Nursing, S.N. College of Nursing, Sri Ganganagar.
}

\section{ABSTRACT}

Corresponding Email: dr.ssoni0201@gmail.com

Introduction: Being a mother is a daily decision to make the other person happy and well-being and to teach the hard lesson of doing the right thing and to repeatedly excuses ourselves for doing anything wrong.

Methodology: study was conducted at S.N. College of Nursing 4 ML Sri Ganganagar Rajasthan. 120 B Sc third year Student Nurses were selected as a sample using a randomized testing procedure. The data was collected by structured questionnaire and analyzed by using descriptive and non-descriptive statistics.

Result: Only 3\% of students with adequate knowledge of $\mathrm{KMC}$ accounted for $64 \%$ in the third year B Sc student nurse with sufficient knowledge of KMC and 33\% in the third year B Sc (Nsg.)and adequate knowledge regarding KMC during the initial post test examination of only $74 \%$ B Sc student nurse with sufficient knowledge regarding Kangaroo care 26\% third year B Sc student nurse with equally sufficient knowledge regarding $\mathrm{KMC}$ and $0 \%$ third year $\mathrm{B} \mathrm{Sc}$ Students Nurses with sufficient knowledge of KMC at the third annual B Sc The Students Nurses were 24.1 higher than the previous known knowledge points of 16.73 points for standard deviation postings were 2.63 and the standard post error check was 2.15 and the calculated $t$ number was 21.66 indicating that the definition difference found in the information center was a real difference. Educational interventions in relation to KMC during the third year B Sc Students Nurses this shows that educational interventions regarding KMC have resulted in increased awareness within the third year B Sc student nurses which is why the null hypothesis has been rejected and the research hypothesis $\mathrm{H}_{1}$ was adopted. 


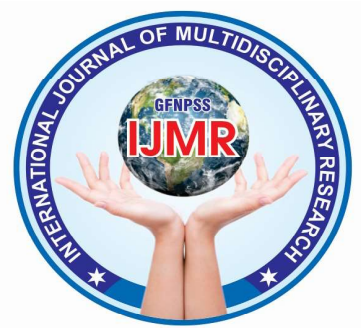

Conclusion: The overall pre test mean 16.73 and post test mean 24.1 the post test mean was more than the pre test mean the " $\mathrm{t}$ " test values is 21.66. So the educational intervention on KMC is very effective among B Sc (Nsg.) students.

Keyword: Effectiveness, Awareness programme, Kangaroo mother care

\begin{tabular}{ccc}
\hline Received & Accepted & Available online \\
\hline $07 / 05 / 2021$ & $15 / 05 / 2021$ & $01 / 06 / 2021$ \\
\hline
\end{tabular}

\section{INTRODUCTION}

A mother's love is the spark that makes the average person able to make this impossible. ${ }^{1}$

The mother plays an important role as the heart of the home but this does not teach the equally important role that the father should play as the head of the household in raising and training their children. ${ }^{2}$

Being a mother is a decision to make every day to put someone else's happiness and priority and to teach them the difficult lesson of doing the right thing and forgiving themselves and doing wrong. ${ }^{3}$

KMC began in 1978 in Bagota Colombia to compensate for the lack of medical equipment but continued to become a common practice. ${ }^{4}$
$\mathrm{KMC}$ is a form of prenatal care with low birth weight that involves skin-to-skin contact in between the caregiver and infants and can begin immediately after birth. ${ }^{5}$

KMC is defined as a practice in which mature infants are placed directly on the parents' chest such as there is a skin-to-skin contact in between the infant and the mother or the father and the breastfeeding mother. ${ }^{6}$

\section{RESEARCH METHODOLOGY}

Research Approach: - Evaluated approach. Research Design: - Pre experimental one group pre test post test design approach was adopted.

Settings: - Study was conducted in selected Nursing colleges of Sri Ganganagar Rajasthan. 


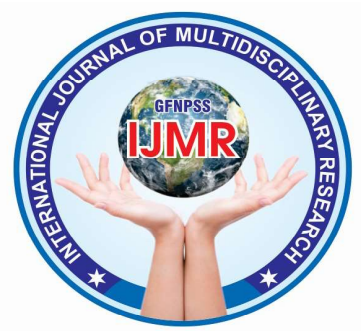

Target Population: - Students those who studying in selected Nursing colleges of Sri Ganganagar Rajasthan.

Accessible Population: - Third year \& Forth year B Sc (Nsg.) student.

Sample: - In this study the sample comprised of 120 B Sc (Nsg.) students.

Sampling Technique: - Random sampling technique.

Sample Size: - 120 B Sc (Nsg.) Student.

\section{RESULTS}

Part A : Analysis of demographic characteristics of the respondents.

Part B: Analysis of the level of knowledge among B Sc Student Nurses regarding kangaroo mother care.

Part C: Analysis of the effectiveness of educational intervention on Kangaroo mother care among B Sc (Nsg.) student.

Part D: Analysis of the impact of demographic variables with mean knowledge score of B Sc Students Nurses regarding Kangaroo mother care.

\section{Part A: Analysis of demographic characteristics of $3^{\text {rd }}$ year B Se (Nsg.) students.}

As shown in sample consists of 120 students of B Sc (Nsg.) and data analysis revealed that $46.66 \%$ were in the age interval of 18 to 20 years and $53.33 \%$ B Sc (Nsg.) student were in the age group of 21 to 23 years and last $0 \%$ was in age group of more than 23 years in B Sc (Nsg.) $76.66 \%$ male and $23.33 \%$ female B Sc Student Nurses 70\% were Hindu and 30\% Sikh all B Sc (Nsg.) student $93.33 \%$ Hindi medium and $6.66 \%$ from CBSE most of the B Sc (Nsg.) Parents $93.33 \%$ were illiterate and $6.66 \%$ were literate most of the B Sc Students Nurses mother occupation is housewife $100 \%$ most of the B Sc Students Nurses fathers occupation $60 \%$ were self $13.33 \%$ were private $20 \%$ were Government employees and $6.66 \%$ were labor most of the $\mathrm{B} \mathrm{Sc}$ Students Nurses $46.66 \%$ were the income group of more than Rs 10000 and $40 \%$ were in the income group of 5000 to 10000 only $13.33 \%$ B Sc Students Nurses were in the income group of 1000 to 2000 majority of B Sc Students Nurses73.33\% were from rural area only $26.66 \%$ urban B Sc Students Nurses $66.33 \%$ were from nuclear family and $33.33 \%$ were from joint family and $3.33 \%$ from extended family majority of B 


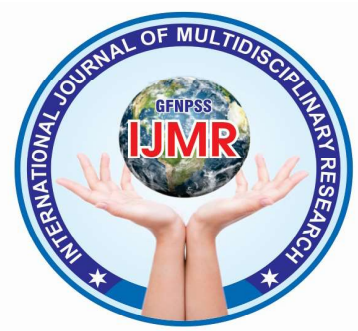

Sc Students Nurses $90 \%$ were from des

deviation was 2.15 and the pre test standard only $10 \%$ were hostler.

Part B: analysis of the level of knowledge deviation of the standard deviation was 2.63 among B Sc Students Nurses regarding kangaroo mother care.

$3 \%$ students of B Sc (Nsg.) with insufficient knowledge regarding Kangaroo maternal care by $64 \%$ B Sc Students Nurses with insufficient knowledge regarding Kangaroo maternal care and 33\% B Sc Students Nurses with insufficient knowledge about Kangaroo maternal care.

Description of post test knowledge score level of B Sc (Nsg.) students.

$74 \%$ B Sc student nurse with sufficient knowledge regarding Kangaroo maternal care $26 \%$ B Sc student nurse with equally sufficient knowledge regarding kangaroo maternal care and $0 \% \mathrm{~B}$ Sc student nurse with insufficient knowledge regarding Kangaroo maternal care after providing educational intervention regarding kangaroo maternal care.

Description of knowledge level of student before and after administration of PTP Mean data after the B Sc the nursing student was 24.1 higher than the pre test data with a standard deviation of 16.73 the standard indicating the effectiveness of educational interventions regarding $\mathrm{KMC}$ during the third year of the B Sc student nurse.

Part C: Analysis of the effectiveness of educational intervention PTP on knowledge of B Sc (Nsg.) student regarding kangaroo mother care.

Pre test and post test knowledge standard error was 0.61 and the calculated $t$ value was 21.66 it shows that the mean difference obtained in the knowledge score was true difference and not by chance suggesting the effectiveness of educational intervention regarding mother care among B Sc Students Nurses this showed that the educational intervention regarding Kangaroo mother care was effective to increasing the knowledge among B Sc Students Nurses hence the null hypothesis was rejected and the research hypothesis $\mathrm{H}_{1}$ was accepted.

Part D: Analysis of the impact of selected demographic variables with mean knowledge score of B Sc Students Nurses regarding kangaroo mother care.

Calculated value of chi square is less than the tabulated value of chi square at 5\% level 


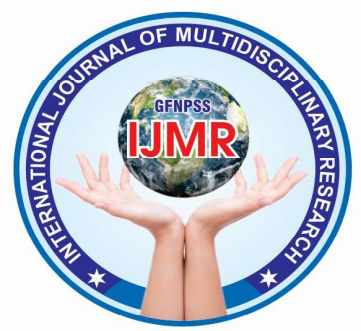

of significance so the researcher had

9. Pre test knowledge score on Kangaroo concluded that:-

There was no association between:mother care and source of information of B Sc (Nsg.) students.

1. Pre test knowledge score on Kangaroo mother care and age of B Sc (Nsg.) students.

2. Pre test score on Kangaroo mother care and sex of B Sc (Nsg.) student.

3. Pre test knowledge score on Kangaroo mother care and education of parents of B Sc (Nsg.) students.

4. Pre test knowledge score on kangaroo mother care and occupation of mother of B Sc (Nsg.) student.

5. Pre test knowledge score on kangaroo mother care and occupation of father of B Sc (Nsg.) students.

6. Pre test knowledge score on Kangaroo mother care and income of family of B Sc (Nsg.) students.

7. Pre test knowledge score on Kangaroo mother care and type of family of B Sc (Nsg.) student.

8. Pre test knowledge on Kangaroo mother care and place of living of B Sc (Nsg.) students.

Hence For above variables hypothesis $\mathrm{H}_{2}$ is rejected.

There was association between the pre test knowledge score on Kangaroo mother care and religion of B Sc (Nsg.) students.

There was association between the pre test knowledge score on Kangaroo mother care and medium of instruction of B Sc (Nsg.) students.

Hence for above variables hypothesis $\mathrm{H}_{2}$ is accepted.

\section{CONCLUSION}

The average test score of 24.1 post-natal post-workout exercises was significantly higher than the best information figures of 16.73 during B Sc Student Nurses calculated the number of $t$ to obtain information points was 21.66. This indicates that educational interventions had improved the knowledge of the B Sc Students Nurses in relation to KMC there was an important measure of interfaith knowledge and the language of instruction and the language of instruction it was concluded that teaching strategies should be developed by the B Sc Student 


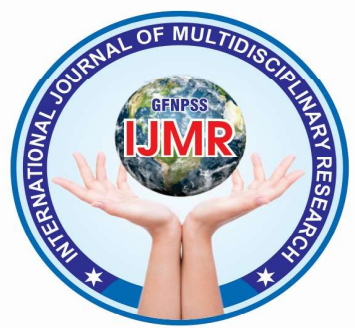

Nurses by improving their knowledge of

IV. Pilliteri A maternal and child health KMC.

\section{BIBLIOGRAPHY}

I. Essential newborn care report of a technical working group World health organization WHO/FRM/MSMM/13.

II. Indian statistics of newborn 2004 www healthstatistic.com.

III. Park k 2007 textbook of preventive and social medicine 19th edition Jabalpur banarsi Das Bhanot ed 19.353-355. nursing care of child Bearing and child bearing family Philadelphia Lippincott company edition 15.2000.698.

V. Price Gwin pediatric nursing $10^{\text {th }}$ edition saunders Elsevier 2008 page number 81 to 82 .

VI. Roberts KL Paynter C A comparison of kangaroo mother care and conventional cuddling care neonatal network June 2004 volume 19431 to 35 . 